\title{
OSCILLATION THEOREMS FOR SECOND-ORDER NONLINEAR DIFFERENTIAL EQUATIONS OF EULER TYPE
}

\author{
James S. W. Wong
}

\begin{abstract}
Consider the second-order nonlinear differential equation

$$
t^{2} x^{\prime \prime}+f(x)=0
$$

where $f(x)$ is continuous and satisfies $x f(x)>0$ if $x \neq 0$, but otherwise no monotonicity condition is imposed. In particular, $f(x)$ is neither sublinear nor superlinear. Here we discuss the oscillation and nonoscillation of solutions of (E) when $f(x)$ is assumed to be asymptotically linear. Results are applicable to the critical case $f(x) / x \rightarrow 1 / 4$ as $|x| \rightarrow \infty$. In particular, when $f(x)=$ $x / 4+\lambda x /(\log |x|)^{2}$, equation (E) is oscillatory if $\lambda>1 / 4$ and non-oscillatory if $\lambda \leq 1 / 16$. This extends recent results of Sugie and Hara which were established by a method of reducing equation (E) to a two dimensional autonomous system of Liénard type.
\end{abstract}

\section{Introduction}

Consider the second-order nonlinear differential equation:

$$
t^{2} x^{\prime \prime}+f(x)=0, \quad t \in[0, \infty),
$$

where $f(x)$ satisfies $x f(x)>0$ if $x \neq 0$, and is locally Lipschitz continuous so that all solutions with arbitrary initial conditions are continuable throughout $[0, \infty)$. A nontrivial solution of (1) is said to be oscillatory if it has arbitrarily large zeros, and is said to be nonoscillatory otherwise. Here we are concerned with conditions on $f(x)$ so that all solutions of (1) are either oscillatory or nonoscillatory, in which case we say equation (1) is oscillatory or nonoscillatory for short. It should be noted, however, that in the absence of Sturm's separation theorem, the nonlinear equation (1) can have both oscillatory and nonoscillatory solutions simultaneously.

When the equation is linear, i.e., $f(x)=k x$, equation (1) becomes the celebrated Euler equation, which is oscillatory if $k>1 / 4$ and is nonoscillatory if $k \leq 1 / 4$. Judging from this, we are led to consider the asymptotic behavior of the ratio

$$
f(x) / x \rightarrow 1 / 4 \text { as }|x| \rightarrow \infty .
$$

The purpose of this paper is to study this problem and re-examine the recent results of Sugie and Hara [10] which have made significant steps toward solving this delicate problem.

Contrary to the existing literature on the second-order nonlinear equation

$$
x^{\prime \prime}+a(t) f(x)=0
$$

where $a(t)$ is a continuously differentiable function which is sometimes referred to as the coefficient function, here equation (1) depicts the coefficient explicitly, i.e., $a(t)=t^{-2}$. On the other hand, most results on the oscillation theory of equation (3) require that the nonlinear function $f(x)$ be monotone, like the special case of the Emden-Fowler equation, which further separates into sublinear and superlinear cases

Received February 14, 1996, revised May 6, 1996. 
depending upon whether $x^{-1} f(x)$ is decreasing or increasing in $x$. For general surveys on oscillation results concerning equation (3), see $[12,14,15]$. Here there is no specific information on $f(x)$ for bounded $x$ except the sign requirement that $x f(x)>0$ if $x \neq 0$. When $a(t)$ is non-negative, the following results on equation (3) are known:

Theorem A. (Waltman [11]) Let $p>1$, and $f(x)$ satisfies

$$
\liminf _{|x| \rightarrow \infty} \frac{|f(x)|}{|x|^{p}}>0 \text {. }
$$

If $a(t) \geq 0$ and satisfies

$$
\int_{0}^{\infty} t a(t) d t=\infty
$$

then equation (3) is oscillatory.

Theorem B. ([Wong [12]) Let $f(x)$ satisfy

If $a(t)$ satisfies

$$
\liminf _{|x| \rightarrow \infty} \frac{|f(x)|}{|x|}>0 .
$$

$$
\int_{0}^{\infty} t^{\lambda} a(t) d t=\infty, \quad \text { for some } \lambda<1,
$$

then equation (3) is oscillatory.

Theorem C. (Wong [12]) Let $f(x)$ satisfy

$$
\liminf _{|x| \rightarrow \infty}|f(x)|>0 \text {. }
$$

If $a(t)$ satisfies

$$
\int_{0}^{\infty} a(t) d t=\infty
$$

then equation (3) is oscillatory.

Theorem D. (Wong [12]) Let $a(t)$ be bounded below by a positive constant. If $f(x)$ satisfies

$$
\liminf _{|x| \rightarrow \infty}\left|\int_{0}^{x} f(u) d u\right|=\infty
$$

then equation (3) is oscillatory.

Clearly, none of the above four theorems is applicable to the problem at hand, since $a(t)=t^{-2}$ fails to satisfy (4), (5) and is not bounded below by a positive constant.

Sugie and Hara [10] studied equation (1) by reducing it to the second-order autonomous system,

$$
\dot{x}=y+x, \quad \dot{y}=-f(x),
$$

where the dot denotes differentiation with respect to $s=\log t$. This reduction is significant in that the resulting autonomous system then can be studied by the methods from the Poincaré-Bendixson theory. Indeed the system (7) is a special case of the more general Lienard system, which has been the subject of intensive study during the past century. Using results from their earlier investigations for such a system (see Hara et al. [3,4], Sugie [9], and Hara and Sugie [5]), they proved in [10] that the following results concerning oscillation and nonoscillation for equation (1). 
Theorem E. (Sugie and Hara [10]) Let $f(x)$ satisfy

$$
\frac{f(x)}{x} \geq \frac{1}{4}+\frac{\lambda}{\log |x|}, \quad \lambda>0,
$$

for $|x|>R$ for a sufficiently large $R$; then equation (1) is oscillatory.

Theorem F. (Sugie and Hara [10]) Let $f(x)$ satisfy

$$
\frac{f(x)}{x} \leq \frac{1}{4}+\frac{\lambda}{(\log |x|)^{2}}, \quad 0<\lambda<\frac{1}{16},
$$

for $|x|>R$ for a sufficiently large $R$; then equation (1) is nonoscillatory.

The above theorems imply the following corollary, which is the desired extension for the linear case of the Euler equation; see $[6,13]$ for further results in the linear case:

Corollary G. Suppose that $f(x)$ is asymptotically linear, i.e.,

$$
\lim _{|x| \rightarrow \infty} \frac{|f(x)|}{|x|}=k \text {. }
$$

Then equation (1) is oscillatory if $k>1 / 4$ and nonoscillatory if $k<1 / 4$.

We are interested in alternative, and perhaps more direct, proofs of these results since phase-plane analysis for autonomous systems can be cumbersome at times. We also believe that results in oscillation theory for second-order equations can best be developed based on Sturm's theory, rather than Poincare-Bendixson theory. This paper is the outcome of our investigations. We shall give our results for the more general equation (3), thereby extending Theorems $\mathrm{E}$ and $\mathrm{F}$.

\section{Oscillation theorem}

In this section, we shall prove the following extension of Theorem E:

Theorem 1. Assume that $a(t)$ satisfies

$$
a(t) t^{2} \geq 1,
$$

for $t$ sufficiently large, and there exists $R>0$ such that for $|x| \geq R$,

$$
\frac{f(x)}{x} \geq \frac{1}{4}+\frac{\lambda}{(\log |x|)^{2}}, \quad \lambda>\frac{1}{4} .
$$

Then equation (1) is oscillatory.

Note that condition (12) is considerably weaker than (8) in Theorem E.

Proof. Let $x(t)$ be a nonoscillatory solution of (3). Without loss of generality, let $x(t)>0$ for $t \geq t_{0}>0$. Since $a(t) \geq 0$ implies $x^{\prime \prime}(t) \leq 0, x^{\prime}(t)$ must be positive. Otherwise, if $x^{\prime}\left(t_{1}\right) \leq 0$ for some $t_{1}>t_{0}$, then $x(t)$ either tends to minus infinity or $x(t) \equiv$ constant. The first instance clearly contradicts the assumption that $x(t)>0$, for $t \geq t_{0}$. On the other hand, that $x(t)$ is identically constant and satisfies equation (3) implies either $a(t) \equiv 0$ which contradicts (11), or in view of the sign restriction that $x f(x)>0$ if $x \neq 0, x(t) \equiv 0$, which again contradicts $x(t)>0$ for $t \geq t_{0}$.

Since $x(t)>0$ and $x^{\prime}(t)>0$ on $t \geq t_{0}$, then

$$
\lim _{t \rightarrow \infty} x(t)=L>0
$$


exists, which can be infinite. Assume that $L$ is finite; thus by continuity of $f(x)$

$$
\lim _{t \rightarrow \infty} f(x(t))=f(L) .
$$

Integrating (3), we have for $t>s$,

$$
x^{\prime}(t)-x^{\prime}(s)+\int_{s}^{t} a(\tau) f(x(\tau)) d \tau=0
$$

which together with $x^{\prime}(t)>0$ yields

$$
x^{\prime}(s) \geq \int_{s}^{\infty} a(\tau) f(x(\tau)) d \tau
$$

We now integrate (13) once again and by a change of order of integration, we find for $t>T \geq t_{0}$

$$
\begin{aligned}
x(t)-x(T) & \geq \int_{T}^{t} \int_{s}^{\infty} a(\tau) f(x(\tau)) d \tau d s \\
& =\int_{T}^{t}(\tau-T) a(\tau) f(x(\tau)) d \tau+\int_{t}^{\infty}(t-T) a(\tau) f(x(\tau)) d \tau \\
& \geq \int_{T}^{t}(\tau-T) a(\tau) f(x(\tau)) d \tau .
\end{aligned}
$$

Note that if $\tau \geq 2 T, \tau-T \geq \tau / 2$, then (14) can be further estimated as follows:

$$
x(t) \geq \frac{1}{2} \int_{2 T}^{t} \tau a(\tau) f(x(\tau)) d \tau .
$$

For sufficiently large $T$, we also have $f(x(t)) \geq 1 / 2 f(L)$, for $t \geq 2 T$.

Using (11) in (15), we obtain

$$
x(t) \geq \frac{1}{4} \int_{2 T}^{t} \frac{f(L)}{\tau} d \tau,
$$

which diverges as $t \rightarrow \infty$. This contradicts $L$ being finite, hence $\lim _{t \rightarrow \infty} x(t)=\infty$ monotonically.

We therefore can choose $t_{2} \geq t_{1} \geq t_{0}$ sufficiently large so that $x(t) \geq R$ for $t \geq t_{2}$, so that (12) becomes applicable. Since $x^{\prime \prime}(t) \leq 0$, so $x(t) \leq x\left(t_{2}\right)+x^{\prime}\left(t_{2}\right)\left(t-t_{2}\right) \leq K t$ for some suitable positive constant $K$. Note that for $\varepsilon>0$, there again exists $t_{3} \geq t_{2}$ so that for $t \geq t_{3}$,

$$
\log x(t) \leq \log K+\log t \leq(1+\varepsilon) \log t .
$$

Using (16) in (12), we have

$$
\frac{a(t) f(x)}{x} \geq \frac{f(x)}{t^{2} x} \geq \frac{1}{4 t^{2}}+\frac{\lambda}{(1+\varepsilon)^{2}(\log t)^{2} t^{2}} .
$$

Choose $\varepsilon$ so small that $\lambda>(1+\varepsilon)^{2} / 4$, which is possible since $\lambda>1 / 4$. Simply choose $0<\varepsilon<2 \sqrt{\lambda}-1$.

Now compare equation (3) with the linear equation

$$
y^{\prime \prime}+\left(\frac{1}{4 t^{2}}+\frac{\lambda}{(1+\varepsilon)^{2}(\log t)^{2} t^{2}}\right) y=0,
$$

which is oscillatory if $\lambda>(1+\varepsilon)^{2} / 4$. By Sturm's Comparison Theorem, we conclude that equation (3) is oscillatory. The proof is complete. 


\section{Nonoscillation theorem}

In this section, we consider a similar extension of Theorem $\mathrm{F}$ for equation (3), namely in the case when all solutions of (3) are nonoscillatory. Judging from the nonoscillation theorems for equation (3), when $f(x)=x|x|^{\gamma-1}, \gamma>0$, see e.g., Heidel [2], Gollwitzer [1], Wong [16], Kwong and Wong [8], it becomes clear that a certain condition on the derivative of the coefficient $a(t)$ would be necessary, and in most cases, the requirement is that $a(t)$ should decrease "monotonically" to zero in a certain way. Since we only require $f(x)$ to behave linearly for large $x$, we need to impose more "stringent" assumptions on the coefficient $a(t)$; although it is not known whether such a condition involving $a^{\prime}(t)$ indeed is necessary. The following result extends Theorem $\mathrm{F}$ to equation (3).

Theorem 2. Assume that $a(t) \geq 0, a(t) \in C^{1}[0, \infty)$, and satisfies

$$
a(t) t^{2} \leq 1
$$

for $t$ sufficiently large, and

$$
A(t)=\frac{a^{\prime}(t)}{2 a^{3 / 2}(t)}+1=o(1), \quad \text { as } t \rightarrow \infty .
$$

If, in addition, $A(t) \leq 0$ and for some large $R>0$ it satisfies

$$
\frac{f(x)}{x} \leq \frac{1}{4}+\frac{\lambda}{(\log |x|)^{2}}, \quad \lambda \leq \frac{1}{16}
$$

then equation (3) is nonoscillatory.

The proof of Theorem 2 is somewhat more complicated than that of Theorem 1. We begin with the following

Lemma. If $x(t)$ is an oscillatory solution of (3), then $x(t)$ is unbounded.

Proof. Define $E(t)$ in terms of $x(t)$ as follows

$$
E(t)=\frac{1}{2}\left(\frac{x^{\prime}}{\sqrt{a}}-x\right)^{2}+F(x)
$$

where $F(x)=\int_{0}^{x} f(u) d u$. Additionally, define

$$
V(t)=\frac{1}{2} \frac{x^{\prime 2}}{a}+F(x) .
$$

Using (3), it is easy to see that $V^{\prime}(t)=-a^{\prime} x^{\prime 2} / 2 a^{2} \geq 0$ since $A(t) \leq 0$.

Thus, if $x(t)$ is oscillatory and bounded, say $|x| \leq B_{1}$, then $V(t)$ is bounded. In fact $V(t) \leq F\left(B_{1}\right)$, and $\left|x^{\prime} / \sqrt{a}\right| \leq B_{2}$ for some suitable constant $B_{2}$. We can relate $E(t)$ to $V(t)$ by $(20)$ and (21) as follows

$$
E(t)=V(t)+\frac{x^{2}}{2}-\frac{x^{\prime} x}{\sqrt{a}}
$$

from which we deduce that

$$
E(t) \leq F\left(B_{1}\right)+\frac{B_{1}^{2}}{2}+B_{1} B_{2}
$$

for all $t$. We now shall show that $E(t)$ bounded is incompatible with the rest of the hypothesis of Theorem 2 . Use (3) to compute $E^{\prime}(t)$

$$
E^{\prime}(t)=-\frac{A{x^{\prime}}^{2}}{\sqrt{a}}+A x x^{\prime}+\sqrt{a} x f(x) \text {. }
$$


Recall that $V^{\prime}(t) \geq 0$, so if $x(t)$ is bounded and oscillatory, then $V_{\infty}=\lim V(t)$ exists, and $V_{\infty}$ is positive and finite. Choose $\varepsilon_{0}$ sufficiently small so that $V_{\infty}>F\left(\varepsilon_{0}\right)+\varepsilon_{0}^{2}$. Hence, for sufficiently large $t$ and for $|x(t)| \leq \varepsilon_{0}$, we have

$$
\frac{1}{2} \frac{x^{\prime 2}}{a} \geq V_{\infty}-F\left(\varepsilon_{0}\right)-\frac{\varepsilon_{0}^{2}}{2}>\frac{\varepsilon_{0}^{2}}{2}
$$

Thus $x^{\prime 2} / a \geq \varepsilon_{0}^{2} \geq|x|^{2}$, from which it follows that $x^{\prime}\left(x^{\prime} / \sqrt{a}-x\right) \geq 0$ for all $t$ when $|x(t)| \leq \varepsilon_{0}$. Define for $\varepsilon_{0}>0$, the following positive numbers:

$$
M=\max _{|x| \leq B_{1}} f(x), \quad m_{0}=\min _{\varepsilon_{0} \leq|x| \leq B_{1}} x f(x) .
$$

Rewrite (24) as

$$
E^{\prime}(t)=-A x^{\prime}\left(\frac{x^{\prime}}{\sqrt{a}}-x\right)+\sqrt{a} x f(x) .
$$

Since $A \leq 0$, so for $|x(t)| \leq \varepsilon_{0}$, we have $E^{\prime}(t) \geq 0$. Otherwise, if $|x(t)| \geq \varepsilon_{0}$, then $\left|A x^{\prime}\left(x^{\prime} / \sqrt{a}-x\right)\right|=o(\sqrt{a})$ by $(18)$; thus, there exists $m_{1}>0, m_{1}<m_{0}$, such that for large $t$,

$$
E^{\prime}(t) \geq m_{1} \sqrt{a}
$$

In either case, we have $E^{\prime}(t) \geq 0$ for all $t$ sufficiently large. Using (3) once again, we note that

$$
\begin{aligned}
-\left(\frac{x^{\prime}}{\sqrt{a}}-x\right)^{\prime} & =-\frac{x^{\prime \prime}}{\sqrt{a}}+x^{\prime}\left(\frac{a^{\prime}}{2 a^{3 / 2}}+1\right) \\
& =\sqrt{a}\left(f(x)+\frac{A(t) x^{\prime}}{\sqrt{a}}\right) \leq M_{1} \sqrt{a}
\end{aligned}
$$

for some $M_{1}>M$ since $A(t)=o(1)$ as $t \rightarrow \infty$. Let $\left\{t_{k}\right\}$ be the sequence of zeros of $x(t)$. For $\varepsilon_{0}>0$, there exist $\sigma_{k}, \tau_{k}$ such that $t_{k}<\sigma_{k}<\tau_{k}<t_{k+1}$ so that $x\left(\sigma_{k}\right)=x\left(\tau_{k}\right)=\varepsilon_{0}$. We note from (22) and (23) that $E_{\infty}=\lim _{t \rightarrow \infty} E(t)$ exists and is finite. For sufficiently large $k, \frac{1}{2}\left(x^{\prime} / \sqrt{a}\left(\sigma_{k}\right)-\varepsilon_{0}\right)^{2} \geq E_{\infty}-2 F\left(\varepsilon_{0}\right)=K_{0}$. Likewise, we have $\frac{1}{2}\left(x^{\prime} / \sqrt{a}\left(\tau_{k}\right)-\varepsilon_{0}\right)^{2} \geq K_{0}$. We can choose subsequences in $\left\{\sigma_{k}\right\}$ and $\left\{\tau_{k}\right\}$, if necessary, and obtain

$$
\frac{x^{\prime}\left(\sigma_{k}\right)}{\sqrt{a}}-\varepsilon_{0} \geq \sqrt{2} K_{0}, \quad \frac{x^{\prime}\left(\tau_{k}\right)}{\sqrt{a}}-\varepsilon_{0}<-\sqrt{2} K_{0} .
$$

Using (27) and integrating (26), we find

$$
\left(\frac{x^{\prime}\left(\sigma_{k}\right)}{\sqrt{a}}-\varepsilon_{0}\right)-\left(\frac{x^{\prime}\left(\tau_{k}\right)}{\sqrt{a}}-\varepsilon_{0}\right) \leq M_{1} \int_{\sigma_{k}}^{\tau_{k}} \sqrt{a}
$$

from which it follows

$$
2 \sqrt{2} K_{0} \leq M_{1} \int_{\sigma_{k}}^{\tau_{k}} \sqrt{a}
$$

Returning to (25) and integrating from $\sigma_{0}$ to $\tau_{k}$, we have by (28)

$$
\begin{aligned}
E\left(\tau_{k}\right)-E\left(\sigma_{0}\right) & \geq \int_{\sigma_{0}}^{\tau_{k}} E^{\prime}(t) d t \geq \sum_{j=0}^{k} \int_{\sigma_{j}}^{\tau_{j}} E^{\prime}(t) d t \\
& \geq \sum_{j=0}^{k} m_{0} \int_{\sigma_{j}}^{\tau_{j}} \sqrt{a} \geq k m_{0} 2 \sqrt{2} K_{0} / M_{1}
\end{aligned}
$$


which tends to infinity as $k \rightarrow \infty$. This contradicts the fact that $E(t)$ is bounded. Hence, $x(t)$ must be unbounded.

Turning to the proof of Theorem 2, let $x(t)$ be an oscillatory solution of (3). The above lemma shows that $x(t)$ is unbounded, so there exists sequences $\left\{\xi_{k}\right\},\left\{\eta_{k}\right\}, t_{k}<$ $\xi_{k}<\eta_{k}<t_{k+1}$ such that $x\left(\xi_{k}\right)=x\left(\eta_{k}\right)=R$ and $x(t)>R$ for $\xi_{k}<t<\eta_{k}$. Denote $I_{k}=\left[\xi_{k}, \eta_{k}\right]$. We shall now apply a Wronskian-type argument to equation (3) on $I_{k}$. In fact, we do this through a transformed equation. Let $s=\log t, u(s)=x(t) / \sqrt{t}$, then equation (3) becomes

$$
\ddot{u}(s)+u(s)\left(\frac{t^{2} a(t) f(x)}{x}-\frac{1}{4}\right)=0 .
$$

For $s=\log t, t \in I_{k}$, we have $x(t)>R$. Now using (17) and (19) in (29), we have

$$
\ddot{u}(s)+\frac{\lambda}{(\log |x|)^{2}} u(s) \geq 0 .
$$

Note that $x(t)=\sqrt{t} u(s), \log |x|=\frac{1}{2} \log t+\log u(s)$, and $\dot{u}(s)=\frac{1}{\sqrt{t}}\left(t x^{\prime}-x / 2\right)$. Since $E(t) \uparrow \infty$ implies $E\left(\xi_{k}\right)=\frac{1}{2}\left(x^{\prime}\left(\xi_{k}\right) / \sqrt{a}\left(\xi_{k}\right)-R\right)^{2}+F(R) \rightarrow \infty$, hence $x^{\prime}\left(\xi_{k}\right) / \sqrt{a}\left(\xi_{k}\right)-$ $R \uparrow \infty$. We now integrate (18) to obtain

$$
-\frac{1}{\sqrt{a}}+\frac{1}{\sqrt{a\left(t_{0}\right)}}+t=o(1)
$$

or $1 / \sqrt{a}=t+o(t)$. Thus, $x^{\prime}\left(\xi_{k}\right) / \sqrt{a}\left(\xi_{k}\right) \rightarrow \infty$ implies $\xi_{k} x^{\prime}\left(\xi_{k}\right) \rightarrow-\infty$ as $k \rightarrow \infty$. For $\xi_{k}$ sufficiently large, $\xi_{k} x^{\prime}\left(\xi_{k}\right)>R$, so $\dot{u}\left(\log \xi_{k}\right)>0$. On $I_{k}=\left[\xi_{k}, \eta_{k}\right]$, there exists $\zeta_{k} \in I_{k}, \xi_{k}<\zeta_{k}<\eta_{k}$ such that $\dot{u}\left(\log \zeta_{k}\right)=0$. This is possible because for $\xi_{k}<s_{k}<\eta_{k}$ such that $x^{\prime}\left(s_{k}\right)=0$, we have

$$
x^{\prime}\left(s_{k}\right)=0 \Rightarrow \dot{u}\left(\log s_{k}\right)=\frac{-1}{\sqrt{s_{k}}} \frac{x\left(s_{k}\right)}{2}<0 .
$$

In fact, on $I_{k}$, we have $\xi_{k}<\zeta_{k}<s_{k}<\eta_{k}$. Let $J_{k}=\left[\log \xi_{k}, \log \zeta_{k}\right]$ be an interval in the $s$ variable so that $\dot{u}(s) \geq 0$ on $J_{k}$ and

$$
u(s) \geq u\left(\log \xi_{k}\right)=\frac{R}{\sqrt{\xi_{k}}}, \quad s \in J_{k} .
$$

Note that

$$
\begin{aligned}
\log |x| & =\frac{1}{2} \log t+\log u(s) \\
& \geq \frac{s}{2}+\log u\left(\log \xi_{k}\right) \\
& =\frac{s}{2}+\log R-\frac{1}{2} \log \xi_{k} \\
& =\frac{s}{2}+\sigma_{k}
\end{aligned}
$$

where $2 \sigma_{k}=2 \log R-\log \xi_{k}$. Thus on $J_{k}, u(s)$ satisfies

$$
\ddot{u}(s)+\frac{4 \lambda}{\left(s+2 \sigma_{k}\right)^{2}} u \geq 0 .
$$

Compare (31) with the linear equation:

$$
\ddot{\nu}(s)+\frac{1}{4\left(s+2 \sigma_{k}\right)^{2}} \nu=0
$$


which is known to be nonoscillatory, and its general solution in fact can be represented as

$$
\nu(s)=c_{1}\left(s+2 \sigma_{k}\right)^{1 / 2}+c_{2}\left(s+2 \sigma_{k}\right)^{1 / 2} \log \left(s+2 \sigma_{k}\right) .
$$

Differentiating (33), we obtain

$$
\dot{\nu}(s)=\left(\frac{c_{1}}{2}+c_{2}\right)\left(s+2 \sigma_{k}\right)^{-1 / 2}+\frac{c_{2}}{2}\left(s+2 \sigma_{k}\right)^{-\frac{1}{2}} \log \left(s+2 \sigma_{k}\right) .
$$

We note from (34) that $\dot{\nu}(s)$ is positive if $c_{2}>0$ and for $s$ sufficiently large. Choose $\nu(s)$ by the initial conditions

$$
\nu\left(\xi_{k}\right)=u\left(\log \xi_{k}\right) \quad \text { and } \quad \dot{\nu}\left(\log \xi_{k}\right)=\dot{u}\left(\log \xi_{k}\right) .
$$

We then obtain the following system of equations in terms of $c_{1}$ and $c_{2}$,

$$
\begin{aligned}
& \nu\left(\log \xi_{k}\right)=c_{1}(2 \log R)^{1 / 2}+c_{2}(2 \log R)^{1 / 2} \log (2 \log R)=\frac{R}{\sqrt{\xi_{k}}} \\
& \dot{\nu}\left(\log \xi_{k}\right)=\left(\frac{c_{1}}{2}+c_{2}\right)(2 \log R)^{-1 / 2}+\frac{c_{2} \log (2 \log R)}{2(2 \log R)^{1 / 2}}=\frac{\xi_{k} x^{\prime}\left(\xi_{k}\right)-\frac{R}{2}}{\sqrt{\xi_{k}}} .
\end{aligned}
$$

Writing $2 \log R=Q^{2}$ for short, we find

$$
\begin{aligned}
\dot{\nu}\left(\log \xi_{k}\right) & =\left(\frac{c_{1}}{2}+c_{2}\right) Q^{-1}+\frac{c_{2} \log Q}{2 Q} \\
& =\dot{u}\left(\log \xi_{k}\right)=\frac{\xi_{k} x^{\prime}\left(\xi_{k}\right)-R / 2}{\sqrt{\xi_{k}}} .
\end{aligned}
$$

Solving (35) for $c_{2}$, we obtain

$$
c_{2}=\Delta^{-1}\left|\begin{array}{cc}
Q & R / \sqrt{\xi_{k}} \\
1 / 2 Q & \left(\xi_{k} x^{\prime}\left(\xi_{k}\right)-R / 2\right) \sqrt{\xi_{k}}
\end{array}\right|
$$

where

$$
\Delta=\left|\begin{array}{cc}
Q & Q \log Q \\
Q / 2 & 1 / 2 Q+\log Q / 2 Q
\end{array}\right|=1
$$

So, $c_{2}=Q / \sqrt{\xi_{k}}\left(\xi_{k} x^{\prime}\left(\xi_{k}\right)-R / 2\right)-R / 2 Q \sqrt{\xi_{k}}=Q / \sqrt{\xi_{k}}\left(\xi_{k} x^{\prime}\left(\xi_{k}\right)-R / 2-R / 2 Q^{2}\right)$. If $Q>1$, then $c_{2}>\frac{Q}{\sqrt{\xi_{k}}}\left(\xi_{k} x^{\prime}\left(\xi_{k}\right)-R\right)>0$. Thus, for sufficiently large $k, \nu(s)>0$, and also

$$
\dot{u}(s)>0 \text { and } \dot{\nu}(s)>0 \text { on } J_{k} .
$$

Recall that $\lambda \leq 1 / 16$, so by (31) and (32) and the fact that $u\left(\log \xi_{k}\right)=\nu\left(\log \xi_{k}\right)$, we obtain

$$
\ddot{u}\left(\log \xi_{k}\right) \geq \ddot{\nu}\left(\log \xi_{k}\right),
$$

and so $u(s) \geq \nu(s)$ in the right neighborhood of $\log \xi_{k}$. Since $u\left(\log \xi_{k}\right)=u\left(\log \eta_{k}\right)$, it follows that $\nu(s)$ must cross $u(s)$ at some point $\gamma \in\left[\log \xi_{k}, \log \eta_{k}\right]$. We now denote the $s$-interval by $L_{k}=\left[\log \zeta_{k}, \log \eta_{k}\right]$ and consider $\gamma \in J_{k}$ and $\gamma \in L_{k}$ separately.

Let $W(u, \nu)=\dot{u} \nu-\dot{\nu} u$. Note that

$$
W\left(\log \xi_{k}\right)=0 \quad \text { and } \quad \dot{W}=\ddot{u} \nu-\ddot{\nu} u \geq 0 .
$$

If $\gamma \in J_{k}$, then $\dot{\nu}(\gamma)>\dot{u}(\gamma)$ (otherwise $\nu(s)<u(s)$ on $J_{k}$, so there is no crossing), hence

$$
W(\gamma)=u(\gamma)(\dot{u}(\gamma)-\dot{\nu}(\gamma))<0
$$


which contradicts $\dot{W} \geq 0, W\left(\log \xi_{k}\right)=0$. In the second case when $\gamma \in L_{k}$, we also have

$$
\dot{\nu}(\gamma)>0, \quad \dot{u}(\gamma)<0, \quad \text { so again } \quad \dot{\nu}(\gamma)>\dot{u}(\gamma),
$$

and the same argument applies. This contradiction on an interval $J_{k}$ for some large $k$ establishes the fact that $u(s)$ must not be oscillatory. Hence, $x(t)$ is nonoscillatory, and the proof is complete.

\section{Examples and remarks}

(i) We now consider a special case of equation (1) for

$$
f(x)=k x+\frac{\lambda x}{(\log |x|)^{2}}
$$

when $|x| \geq R$ for some large $R>0$. Theorems 1 and 2 show that equation (1) is oscillatory when $k>1 / 4$ and nonoscillatory when $k<1 / 4$. In the critical case when $k=1 / 4$, it is nonoscillatory if $\lambda \leq 1 / 16$. These results improve those obtained by Sugie and Hara [10]. In case of oscillation, their result is not applicable when $k=1 / 4$ since they require that the second term $\lambda / \log |x|$ is large compared to $\lambda /(\log |x|)^{2}$ in (36) above. In case of nonoscillation, their result requires that $\lambda<1 / 16$ as compared to $\lambda \leq 1 / 16$. We conjecture that when $k=1 / 4$ and $1 / 16<\lambda \leq 1 / 4$, equation (1) has both oscillatory and nonoscillatory solutions. However, we are unable to establish results in this direction and hope to return to this problem in a later paper.

(ii) It is of interest to note that the sign requirement $x f(x)>0$ is implicit in condition (12) of Theorem 1. On the other hand, Theorem 2 without the sign requirement is false. Consider

$$
x^{\prime \prime}+\frac{1}{4 t^{2}}\left(x+2 \sqrt{1-x^{2}}\right)=0,
$$

which has $x(t)=\sin \frac{1}{2} \log t$ as an oscillatory solution. Here $f(x)$ is not yet defined for $|x|>1$, so condition (19) in Theorem 2 can be satisfied trivially

(iii) We also note that the Euler-Kneser-types of conditions are known to be valid for oscillations of equation (3). In the linear case, see $[6,13]$, and for superlinear and sublinear cases, see [17]. A similar type of result was given for a delayed differential equation in a recent paper of Kulenovic [7].

(iv) We remark that in conditions (11) and (17), the lower and upper bounds for $t^{2} a(t)$ can in fact be any positive number $\beta$ instead of 1 , since we can always redefine

$$
\bar{a}(t)=\beta^{-1} a(t), \quad \bar{f}(x)=\beta f(x)
$$

and apply results to equation (3) with $\bar{a}(t), \bar{f}(x)$ instead of $a(t), f(x)$.

(v) We also remark that condition (18) in Theorem 2 is undesirable as it implies that $a(t)=t^{-2}+o\left(t^{-2}\right)$ as $t \rightarrow \infty$. This would exclude cases when $a(t)$ is known to be small, say $a(t)=t^{-3}$, in which case it is well-known that not only the linear equation is nonoscillatory, but so are all solutions of the Emden-Fowler equation, i.e., (1) when $f(x)=x|x|^{\gamma-1}, 1<\gamma<\frac{3}{2}$.

(vi) Finally, we consider a nonlinear function $f(x)$ defined by

$$
f(x)= \begin{cases}k x\left(1+\sin x^{2}\right) & |x| \leq 1 \\ k x & |x|>1\end{cases}
$$


and $a(t)=t^{-2}+o\left(t^{-2}\right)$. Here the nonlinear function can have rather erratic behaviour when $|x| \leq 1$. Theorems 1 and 2 imply that equation (3) is oscillatory if $k>1 / 4$ and $a(t)$ satisfies (11) and nonoscillatory if $k<1 / 4$ and $a(t)$ satisfies (17). These conclusions cannot be deduced from any previous known results.

\section{References}

1. M. E. Gollwitzer, Nonoscillatory theorem for a nonlinear differential equation, Proc. Amer. Math. Soc. 26 (1970), 77-84.

2. J. W. Heidel, A nonoscillatory theorem for a nonlinear second order differential equation, Proc. Amer. Math. Soc. 22 (1969), 485-488.

3. T. Hara, T. Yoneyama, and J. Sugie, Continuation results for differential equations by two Liapunov functions, Ann. Mat. Pura. Appl. 133 (1983), 77-92.

4. —_, A necessary and sufficient condition for oscillation of the generalized Lienard equation, Ann. Mat. Pura. Appl. 154 (1989), 223-230.

5. T. Hara and J. Sugie, When all trajectories in the Lienard plane cross the vertical isocline? Nonlinear Differential Equations (to appear).

6. E. Hille, Nonoscillation theorems, Trans. Amer. Math. Soc. 64 (1948), 234-252.

7. M. R. S. Kulenovic, Oscillation of the Euler differential equation with delay, Czech. Math. J. 45 (1995), 1-6.

8. M. K. Kwong and J. S. W. Wong, Nonoscillation theorem for a second order sublinear ordinary differential equation, Proc. Amer. Math. Soc. 87 (1983), 467-474.

9. J. Sugie, The global center for the Lienard system, Nonlinear Analysis, 17 (1991), 33-345.

10. J. Sugie and T. Hara, Nonlinear oscillation of second order differential equations of Euler type, Proc. Amer. Math. Soc. (to appear).

11. P. Waltman, Oscillation of solutions of a nonlinear equation, SIAM Review, 5 (1963), 128-130.

12. J. S. W. Wong, On second order nonlinear oscillation, Funkcialaj Ekvac. 11 (1969), 207-234.

13. - Oscillation and nonoscillation of solutions of second order linear differential equations with integrable coefficients, Trans. Amer. Math. Soc. 144 (1969), 197-215.

14. __ Oscillation theorem for second order nonlinear differential equations, Bull. Ins. Math., Acad. Sinica, 3 (1975), 283-309.

15. _ On the generalized Emden-Fowler equation, SIAM Review 17 (1975), 339-360.

16. __ Remarks on nonoscillation theorems for a second order nonlinear differential equation, Proc. Amer. Math. Soc. 83 (1981), 541-546.

17. _ Oscillation criteria for second order nonlinear differential equations involving integral averages, Can. J. Math. 45 (1993), 1094-1103.

Chinney Investments Ltd, 1218 Swire House, Hong Kong 